\title{
Síndrome compartimental posangioplastía coronaria transradial: A propósito de un caso
}

\author{
Franco Bavestrello R. ${ }^{1}$, Macarena Viedma C. ${ }^{1}$ y Luis Fuentes N. ${ }^{2}$
}

${ }^{1}$ Universidad Andrés Bello Sede Viña del Mar. Chile. Servicio de Cirugía Hospital de Quilpué, Región de Valparaíso. Chile.

Recibido el 8 de septiembre de 2018 y aceptado para publicación el 20 de septiembre de 2018 .

Correspondencia a: Dr. Franco Bavestrello R franco.bavestrello@gmail.com

\section{Post transradial coronary angioplasty compartimental syndrome}

Introduction: The compartment syndrome is caused by an increase on the compartment pressure that exceed the perfusion pressure. In coronary postangioplasty context it is a rare entity. Requires a high suspicion and to take fast steps. Clinical case: Forty-one years old man with a STEMI thrombolysed and derived to angioplasty suffered a post-procedure bleeding on his right forearm and evolved with a compartment syndrome that required a volar and medial fasciotomy. Discussion: The clinical entity is rare in this context, so require a high suspicion based basically on sudden pain with an increase of volume. The treatment is the urgent fasciotomy. To not diagnose it at time could evolve with severe neuromuscular disorders and hand anatomy disorders.

Key words: compartment syndrome; coronary angioplasty; fasciotomy.

\section{Resumen}

Introducción: El síndrome compartimental se genera por aumento de presión compartimental que sobrepasa a la de perfusión. Es una entidad rara en contexto posangioplastía coronaria. Requiere alta sospecha y medidas rápidas. Caso clínico: Hombre de 41 años con diagnóstico de infarto agudo de miocardio (IAM) con supradesnivel ST (SDST) que fue trombolizado y derivado para angioplastía, que sufre sangrado en antebrazo derecho posprocedimiento y evoluciona con síndrome compartimental que requiere de fasciotomía en volar y medial. Discusión: La entidad clínica es rara en este contexto, por ende, requiere alta sospecha basándose principalmente en dolor de inicio súbito con aumento de volumen. El tratamiento es fasciotomía de urgencias. De no pesquisarse a tiempo puede evolucionar con severas alteraciones neuromusculares llevando a trastornos de la anatomía de la mano.

Palabras clave: síndrome compartimental; angioplastía coronaria; fasciotomía.

\section{Introducción}

El síndrome compartimental corresponde a un fenómeno que ocurre cuando la presión de un compartimento fascial sobrepasa la presión de perfusión, llevando a isquemia irreversible y necrosis. Las principales causas que lo producen son fracturas, siendo otras las quemaduras, traumas por aplastamiento o vasculares ${ }^{1}$.

En series de casos grandes, se describe una incidencia de $0,01 \%$ de síndrome compartimental de antebrazo como complicación de la angioplastía transradial, siendo, por ende, una causa muy rara de este fenómeno ${ }^{2}$.

Lo primordial en esta patología es tener una alta sospecha, con el fin de tomar medidas rápidas y evitar complicaciones catastróficas. La principal complicación corresponde al déficit neurológico, cuya secuela disminuye con la descompresión temprana ${ }^{3}$.

\section{Caso clínico}

Paciente de sexo masculino de 41 años con antecedente de HTA en tratamiento con losartán y tabaquismo, que acude a Unidad de Emergencias de Adultos de hospital de baja complejidad por cuadro de dolor torácico irradiado a extremidad superior izquierda, asociado a síntomas neurovegetativos, con electrocardiograma que muestra supradesnivel ST en V1-V5, con elevación de las 
enzimas cardiacas, diagnosticado como IAM con SDST de pared septal. Trombolizado con estreptoquinasa sin incidentes, con criterios de reperfusión clínica y electrocardiográfica. Trasladado a centro de referencia para coronariografía con eventual angioplastía.

Durante procedimiento se constata sangrado de antebrazo derecho con sospecha de síndrome compartimental por aumento de volumen y dolor en la zona. Se deriva a evaluación por cirujano, quien realiza fasciotomía de compartimento anterior.

En posoperatorio mediato, evaluado por cirujano vascular, que constata aumento de volumen y dolor en cara interna de antebrazo derecho e indica fasciotomía de compartimento medial.

Con parestesia de cara medial del dedo índice y en dedo meñique, con movilidad conservada, pulso radial conservado (Figura 1 ).

Se realiza aseo quirúrgico para debridar tejido inviable, con afrontamiento parcial de heridas operatorias (Figura 2).

Evoluciona sin dolor, con disminución de volumen de la zona afectada, sin signos de infección, con buena respuesta a curaciones avanzadas con hidrocoloides, con buena movilidad hacia distal, sin parestesia. Se realiza alta médica con derivación a Unidad de Hospitalización Domiciliaria para continuar curaciones, en espera de eventual injerto de piel para cierre definitivo de heridas operatorias (Figura 3 y 4).

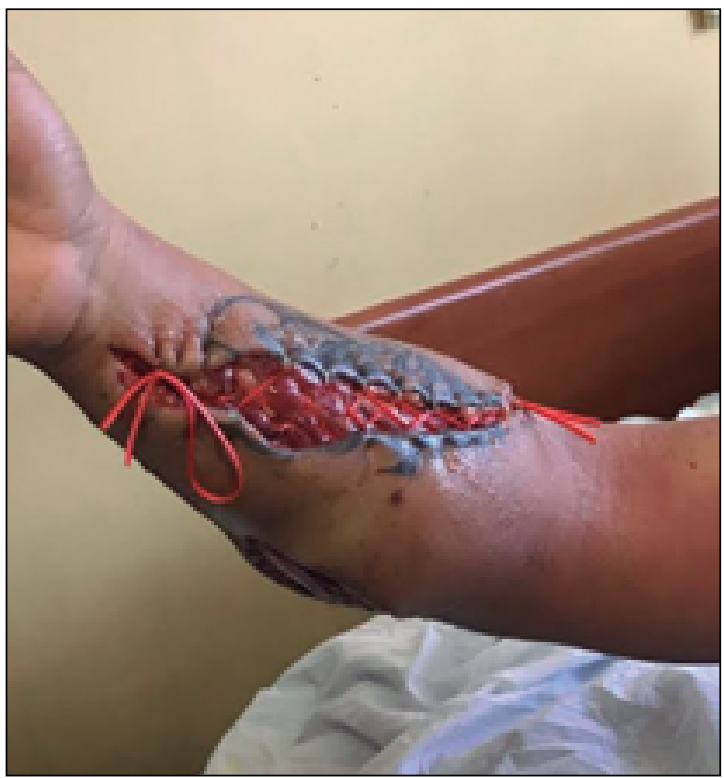

Figura 1. Antebrazo al séptimo día posfasciotomía.

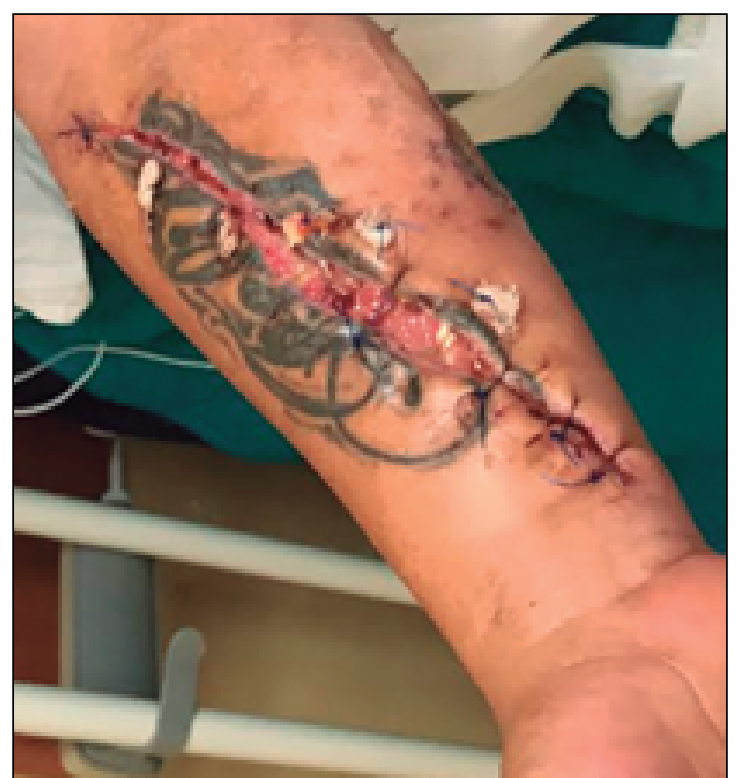

Figura 2. Antebrazo 2 semanas posfasciotomía.

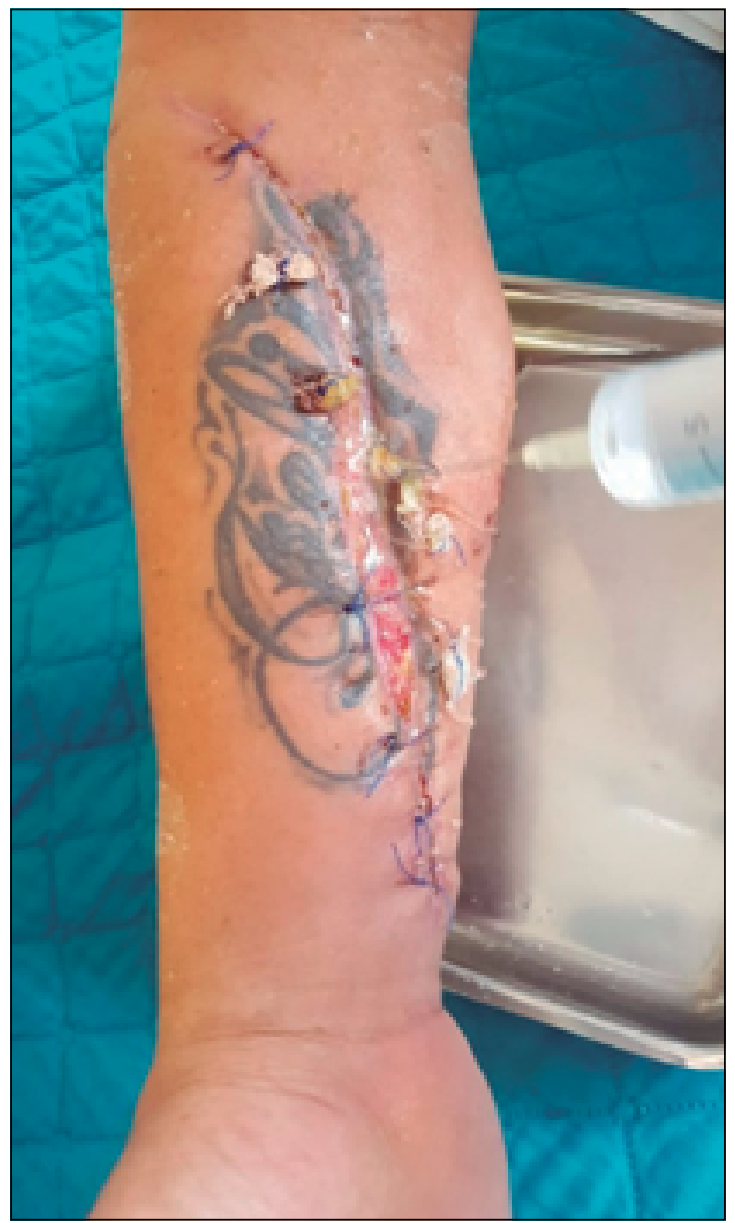

Figura 3. Antebrazo a las 3 semanas posfasciotomía. 
Figura 4. Antebrazo a las 3 semanas posfasciotomía. Compartimento medial.

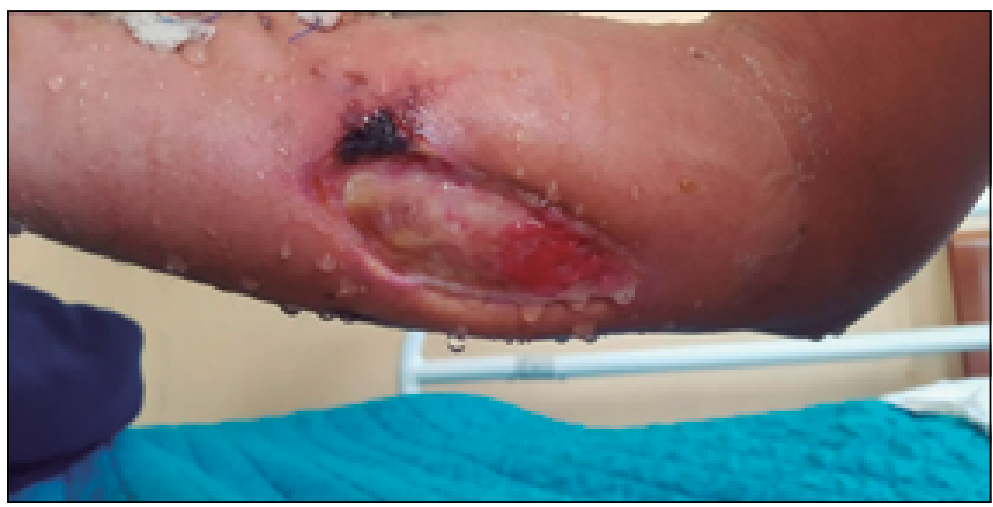

\section{Discusión}

La incidencia de síndrome compartimental relacionado a la cateterización transradial reportada es de un $0,004 \%{ }^{4}$. Algunos metaanálisis han establecido una correlación estadística entre las intervenciones coronarias percutáneas $(\mathrm{PCI})$ y el síndrome compartimental, planteándose como hipótesis el uso de grandes dosis de anticoagulante, que podrían provocar mayor sangrado que provocaría el síndrome compartimental ${ }^{6}$.

Dado las complicaciones asociadas a esta patología, el diagnóstico debe realizarse mediante la clínica y no esperar exámenes de apoyo. Dentro de las características clínicas más importantes se encuentran las "5 P's" (pain, pallor, painful stretching of muscles, paresthesia, and pulselessness); dolor, palidez, contracción muscular dolorosa, parestesia y ausencia de pulsos. El diagnóstico debe realizarse previo a la aparición de todas las P's $\mathrm{s}^{5,7}$.

De no ser resuelto el cuadro, evoluciona a pérdida de la fuerza muscular, posición típica de la mano caracterizada por posición supina, flexión de la muñeca, extensión metacarpofalángica, y flexión interfalángica), seguido de disestesia y pérdida de pulsos. La isquemia provocada a largo plazo genera daño neuromuscular llevando a una contractura de Volkmann, caracterizada en su forma más severa por una mano en garra o flexión interfalángica, extensión metacarpofalángica y atrofia de la musculatura intrínseca de la mano que se producirá por isquemia muscular prolongada, lo cual llevará a necrosis de miocitos y recambio por miofibroblastos que poseen contracción mantenida ${ }^{5,8}$.

Ante el diagnóstico de síndrome compartimental, se debe proceder a fasciotomía inmediata para descomprimir el compartimento afectado. El abordaje más frecuente es una fasciotomía volar, que automáticamente descomprime el compartimento dorsal. De ser requerido, se debe debridar el tejido no viable de la fasciotomía y programar un eventual cierre en un segundo tiempo operatorio ${ }^{3}$.

\section{Agradecimientos}

A los doctores Luis Fuentes Navarrete y Marcelo Vera Segura por su apoyo y fomento a realizar este trabajo, por su docencia y su eterno espíritu del saber.

\section{Responsabilidades éticas}

Protección de personas y animales. Los autores declaran que para esta investigación no se han realizado experimentos en seres humanos ni en animales.

Confidencialidad de los datos. Los autores declaran que en este artículo no aparecen datos de pacientes.

Conflictos de interés: no hay.

\section{Bibliografía}

1. Garner M, Taylor S, Gausden E, Lyden J. Compartment Syndrome: Diagnosis, Management, and Unique Concerns in the Twenty-First Century. HSSJ 2014;10:143-
52. doi: 10.1007/s11420-014-9386-8.

2. Kanei Y, Kwan T, Nakra NC, Liou M. Transradial Cardiac Catheterization: A Review of Access Site Complications. Catheter Cardiovasc Interv. 2011;78:8406. doi: 10.1002/ccd.22978.
3. Kalyani BS, Fisher BE, Roberts CS, Giannoudis PV. Compartment Syndrome of the Forearm: A Systematic Review. J Hand Surg Am. 2011;36:535-43. doi: 10.1016/j.jhsa.2010.12.007.

4. Omori S, Miyake J, Hamada K, Naka 
N. Compartment Syndrome of the Arm Caused by Transcatheter Angiography or Angioplasty. Orthopedics. 2013;36:e1215. doi: 10.3928/01477447-20121217-31.

5. Tizon-Marcos H, Barbeau GR. Incidence of compartment syndrome of the arm in a large series of transradial approach for coronary procedures. J Interv Cardiol. 2008;21:380-4. doi: 10.1111/j.1540- 8183.2008.00361.x.

6. Zwaan EM, Koopman AG, Holtzer CA, Zijlstra F. Revealing the impact of local access-site complications and upper extremity dysfunction post transradial percutaneous coronary procedures. Neth Heart J. 2015;23:514-24. doi: 10.1007/ s12471-015-0747-9.

7. Chugh SK, Chugh Y, Chugh S. How to tackle complications in radial procedures: Tip and tricks. Indian Heart J. 2015; 67:275-81. doi: 10.1016/j. ihj.2015.05.016.

8. Reurings J, Verhofstad M. The Volkmann Ischemic Contracture of the Forearm is Preventable. Eur J Trauma Emerg Surg. 2007;33:539-44. doi: http://sci-hub. tw/10.1007/s00068-007-6142-5. 